\title{
A case report of an infant after episodes of paroxysmal supraventricular tachycardia with left ventricular thrombosis and cerebral infarction
}

\author{
Dan $\mathrm{Li}^{1,2}$, Jinghui Guo ${ }^{1}$, Xiao-Hui Li $^{1,2}$, Yanmei Liao ${ }^{3}$, Ming-Ming Zhang ${ }^{1}$, Lin Shi ${ }^{1}$, Yao Lin ${ }^{1}$, Yang Liu ${ }^{1}$ \\ ${ }^{1}$ Department of Cardiology, Children's Hospital Capital Institute of Pediatrics, Beijing, China; ${ }^{2}$ Department of Cardiology, Children's Hospital \\ Capital Institute of Pediatrics, Graduate School of Peking Union Medical College, Beijing, China; ${ }^{3}$ Department of Echocardiography, Hami Second \\ People's Hospital, Hami, China \\ Correspondence to: Xiao-Hui Li. Children's Hospital Capital Institute of Pediatrics, Graduate School of Peking Union Medical College, 2 Ya-Bao \\ Road, Chaoyang District, Beijing 100020, China. Email: 1xhmaggie@126.com.
}

\begin{abstract}
Left ventricular thrombosis and cerebral infarction caused by recurrent episodes of paroxysmal supraventricular tachycardia (PSVT) are uncommon in infants. We present the case of a 23-month-old girl whose echocardiography revealed a left ventricular thrombus, which resolved after sinus rhythm was restored. The girl had experienced repeated systemic convulsions and high muscular tension of the left limb accompanied by movement disorder. Soon afterward, cranial magnetic resonance imaging (MRI) revealed scattered lacunar cerebral infarctions in the right lateral ventricle. The symptoms, signs, and laboratory examination satisfied the diagnosis of left ventricular thrombosis and cerebral infarction caused by recurrent episodes of PSVT. The girl was given antiarrhythmic drugs, including propranolol, esmolol, and amiodarone, intracranial pressure decreasing treatment (mannitol), heart and brain cell nutritional therapy, anticoagulant therapy, and her condition gradually improved. This case report highlights the importance of pediatric PSVT patients' clinical management and that more emphasis should be placed on early recognition and prevention of severe complications. Pediatricians should be trained early recognition of the nonspecific clinical manifestations of PSVT, make effectively and quickly diagnosis by electrocardiogram, evaluation of cardiac function and thrombosis by echocardiography, and termination PSVT as rapidly as possible. Thromboprophylaxis therapy might be considered for recurrent episodes of PSVT.
\end{abstract}

Keywords: Paroxysmal supraventricular tachycardia (PSVT); left ventricular thrombosis; infant; cerebral infarction

Submitted Sep 08, 2020. Accepted for publication Dec 31, 2020.

doi: 10.21037/apm-20-1797

View this article at: http://dx.doi.org/10.21037/apm-20-1797

\section{Introduction}

Paroxysmal supraventricular tachycardia (PSVT) is the most common arrhythmia in children; it accounts for $90 \%$ of all pediatric arrhythmias (1), and the incidence is $1 / 25,000$ (1). It is characterized by abrupt onset and termination. Longlasting attacks of PSVT may result in hemodynamic instability, heart failure, and other severe consequences (2). Due to a lack of specific clinical manifestations in infants and young children, diagnosis is often delayed, leading to severe pediatric clinical practice complications. To date, papers have reported left atrial appendage thrombus in two neonates $(3,4)$ and left ventricular thrombosis in a 6-weekold girl after sustained PSVT (5). This case report involved a 23-month-old girl who suffered recurrent episodes of PSVT resulting in left ventricular thrombosis and cerebral infarction. The clinical significance of this case report suggested that echocardiography should be performed in time to evaluate cardiac function and thrombosis, episodes of tachycardia are terminated as soon as possible, and thromboprophylaxis therapy be given for recurrent episodes 


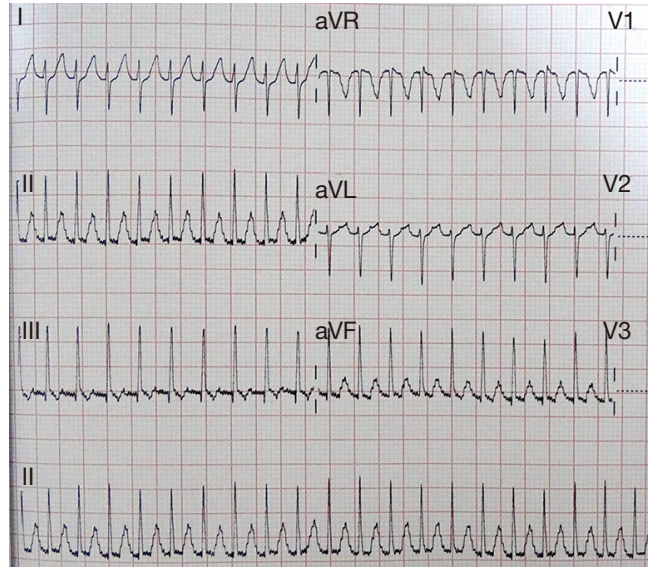

Figure 1 Electrocardiogram showing supraventricular tachycardia.

of PSVT in infants. We present the following case in accordance with the CARE reporting checklist (available at http://dx.doi.org/10.21037/apm-20-1797).

\section{Case presentation}

A 23-month-old girl was admitted to the hospital with the complaint of recurrent episodes of PSVT over a year, and intermittent seizures for eight days. The periodic episodes of PSVT were of 1-2 hours duration each, at a frequency of 3-4 times per week. The attacks occurred during activity or sleep, and were mainly spontaneously terminated. When episodes were of long duration, the girl cried, lost appetite, and became pale. These signs disappeared with termination of PSVT and recovery of sinus rhythm. The girl was given amiodarone and digoxin without good effect. Two weeks before admission, the girl was admitted to the local hospital on continuous crying and pallor. Electrocardiogram (ECG) revealed a ventricular rate of $233 \mathrm{bpm}$ (Figure 1), and echocardiography showed that the motion amplitude of the left ventricular wall was weakened and left cardiac function was decreased [ejection fraction (EF) 44\%]. An abnormal mass, suspected to be a thrombus, was detected in the left ventricle with a small amount of pericardial effusion (Figure $2 A, B$ ). In the process of cardioversion, the girl suffered systemic convulsions and loss of consciousness, and she was subsequently transferred to another hospital. Upon admission to the second hospital, her heart rate was $105 \mathrm{bpm}$ with sinus rhythm (Figure 3). Echocardiography was performed, revealing enlargement of the left ventricle chamber, decreased movement of the ventricular wall (left ventricle diastolic diameter was $29 \mathrm{~mm}$, EF 43\%), and no abnormal mass in the left ventricle. Cranial magnetic resonance imaging (MRI) revealed scattered lacunar cerebral infarctions in the right lateral ventricle. Limb twitching was still present after symptomatic treatment, as were tonic-clonic seizures and muscular hypertonia of the left limb, along with recurrent episodes of PSVT.

The girl was transferred to our hospital for further treatment. The results of physical examination were as follows: blood pressure: left upper extremity $98 / 45 \mathrm{mmHg}$, right upper extremity $92 / 55 \mathrm{mmHg}$, left lower extremity $125 / 68 \mathrm{mmHg}$, right lower extremity $110 / 60 \mathrm{mmHg}$; the patient was alert and active, with smooth respiration, and heart rate $105 \mathrm{bpm}$ with sinus rhythm. The nervous system examination results were as follows: left upper limb tone $3 / 5$, left leg tone $3 / 5$, right arm tone $5 / 5$, right leg tone $5 / 5$. The left Babinski signs (+), the right Babinski signs (-), and Brudzinski's sign (-). The cranial MRI (Figure $4 A$ ) was verified as showing the formation of local cerebral softening lesions and multiple abnormal intracranial signal shadows. During the hospitalization, recurrent episodes of PSVT were observed eight times. Sinus rhythm was maintained by the combination of antiarrhythmic agents, including propranolol, esmolol, and amiodarone, and she was given anti-infective therapy, intracranial pressure decreasing treatment (mannitol), heart and brain cell nutritional therapy, and anticoagulant therapy. The girl's symptoms of infection improved, and anti-infective therapy was stopped accordingly.

Meanwhile, we gradually reduced the treatment of decreasing intracranial conventional pressure until the medication was completely withdrawn. The girl was discharged from our hospital after 22 days and continued with oral propranolol ( $5 \mathrm{mg} / \mathrm{kg} \mathrm{q} 6 \mathrm{~h})$ and amiodarone (3-5 mg/kg/d). Cranial MRI (Figure 4B) was checked 3 weeks after admission, and it revealed that there were liquefaction lesions in the right sub-region of the nucleus, the right frontal lobe, temporal lobe, and occipital lobe; lesions in the left parietal cortex were enhanced, and all lesions had disappeared in diffusion-weighted imaging. Echocardiography (Figure $5 A, B$ ) revealed that atrial and ventricular structure was normal, left ventricle diastolic diameter was $29 \mathrm{~mm}$, and $\mathrm{EF}$ was $66 \%$. In the present case, it is unknown whether the thrombosis was developed by the long-term PSVT alone or atrial fibrillation. During hospitalization, we continuously monitored the patients' ECG, and we did not detect any of the usual characteristics of atrial fibrillation. These physical examinations, clinical manifestations, and imaging results supported the diagnosis 

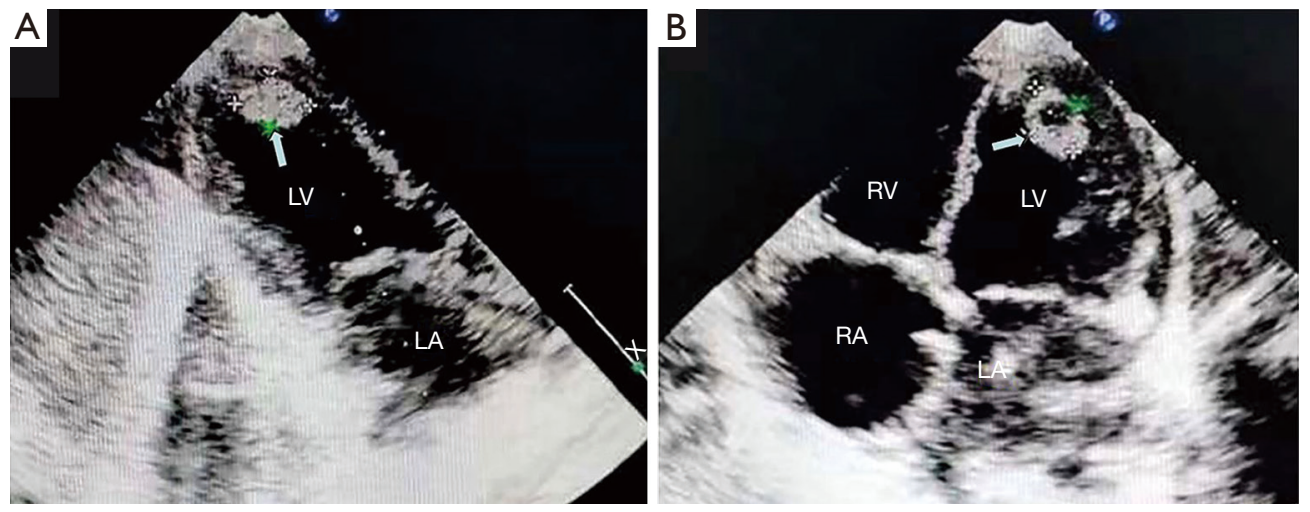

Figure 2 The abnormal echocardiography 2 weeks before admission. (A) Echocardiography showing an abnormal mass (arrow) suspected thrombus in the left ventricle; (B) echocardiography showing an abnormal mass (arrow) suspected thrombus in the left ventricle with a small amount of pericardial effusion. LV, left ventricular; LA, left atrial; RV, right ventricular; RA, right atrial.

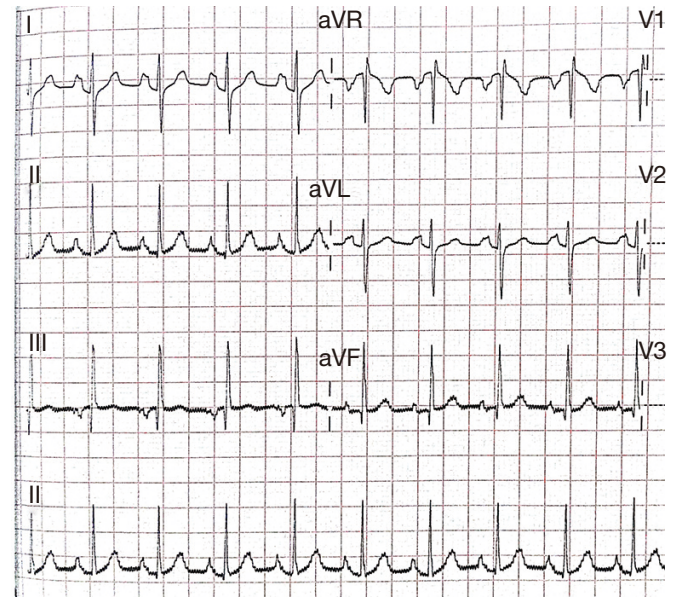

Figure 3 Electrocardiogram showing sinus rhythm.

of left ventricular thrombosis and cerebral infarction.

\section{Follow-up and outcomes}

The patient was followed-up by our cardiology and neurology department, focusing on the liver, kidney, and thyroid function. We performed ECG, echocardiography, thyroid ultrasound, and her left limb and general limb activity were assessed. She was followed-up for 18 months with ECG, echocardiography, and 24-hour dynamic ECG every 3 months. There have been no abnormalities of cardiac size or function and no further attacks of PSVT on drug treatment. No obvious abnormalities were found at the follow-up physical examination. During follow-up, we did not record any adverse or unanticipated events. Doses of medication are currently gradually tapered. (All procedures performed in studies involving human participants were in accordance with the ethical standards of the institutional and/or national research committee(s) and with the Helsinki Declaration (as revised in 2013). This study was approved by the ethical review committee of The Children's Hospital Capital Institute of Pediatrics (registration number: SHERLL2018020). Written informed consent was obtained from the patient for publication of this manuscript and any accompanying images).

\section{Discussion}

Although PSVT is the most common tachyarrhythmia in children, few reports of thrombosis result from PSVT in children. So far, there have only been three reported cases of PSVT causing left atrial appendage and left ventricular thrombosis (3-5) in pediatric patients.

We reported the case of a 23 -month-old girl with echocardiography revealing a thrombus in the left ventricle with heart failure and signs of peripheral embolization (cerebral infarction) following episodic PSVT. When her sinus rhythm was restored, neurological sequelae resulting from cerebral infarction remained, and it was characterized by the increased tone and impaired movement of the left side.

The defining characteristic of PSVT is its abrupt onset and termination, with varying durations (6). Clinical manifestations include anxiety, tachypnea, pallor, cyanosis, and poor appetite. An ECG examination is required for confirmation of PSVT (7-9). Diagnosis and treatment may 

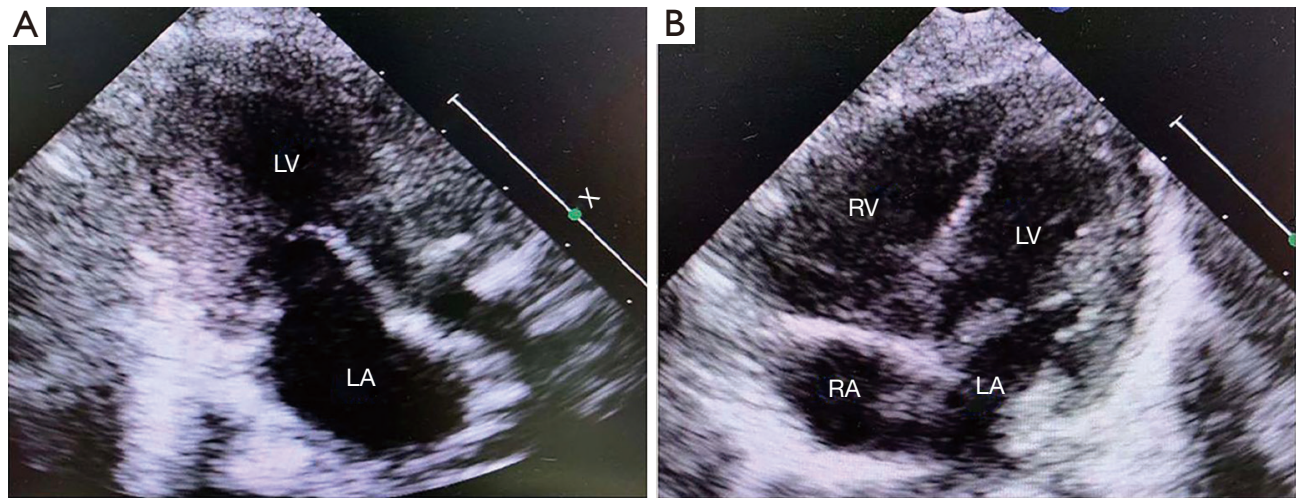

Figure 4 Cranial MRI: the cranial MRI checked during the hospitalization and 3 weeks after admission. (A) The formation of local cerebral softening lesions and multiple abnormal intracranial signal shadows; (B) there were liquefaction lesions in the right sub-region of the nucleus, the right frontal lobe, temporal lobe, and occipital lobe; lesions in the left parietal cortex were enhanced, and all lesions had disappeared in diffusion-weighted imaging. LV, left ventricular; LA, left atrial; RV, right ventricular; RA, right atrial; MRI, magnetic resonance imaging.
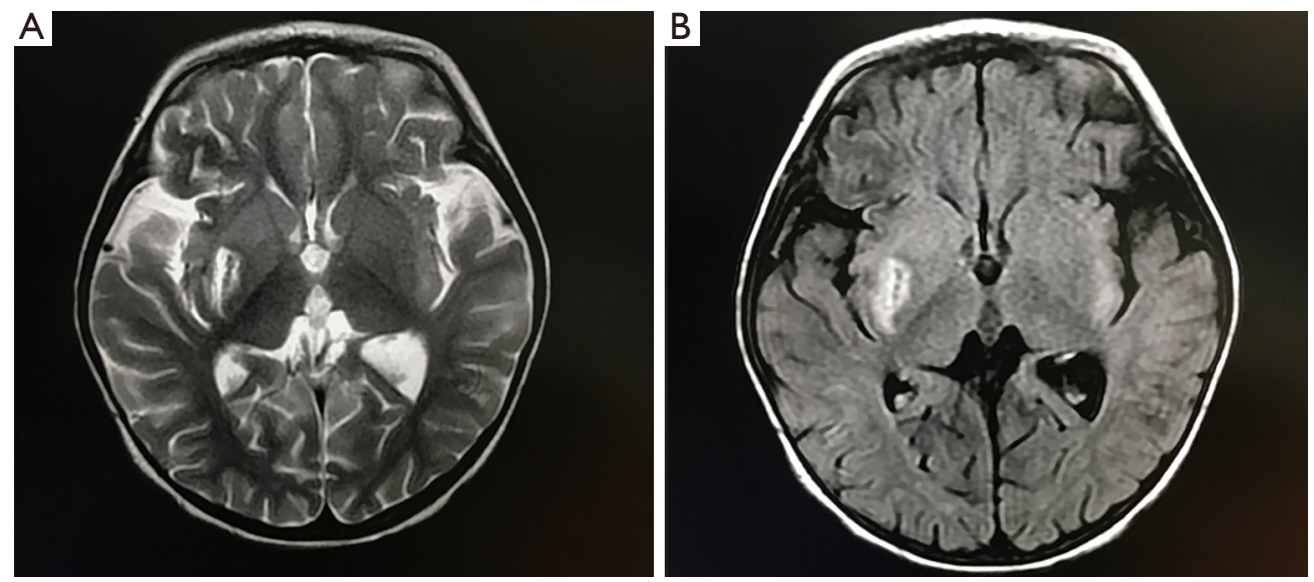

Figure 5 The echocardiography checked 3 weeks after admission. (A) Echocardiography showing the atrial and ventricular structures were normal, no thrombus or pericardial effusion was observed; (B) echocardiography showing the atrial and ventricular structures were normal, no thrombus or pericardial effusion was observed.

be delayed due to nonspecific symptoms in infants or young children and result in severe sequelae. In the present case, the child's episodes of PSVT recurred until she developed serious complications, including ventricular thrombus and cerebral infarction.

When the girl was admitted to our department, she remained in sinus rhythm with intermittent episodes of PSVT. There are multiple possible sources or causes of cerebral infarction, and it becomes difficult to differentially diagnose PSVT as the cause after an episode has terminated. After our team had carefully reviewed the medical history and performed related examinations, no other risk factors, except for PSVT, were found for thrombosis and neurological diseases leading to cerebral infarction in the girl, such as coagulation disorders, hepatic function abnormality, septicemia, disseminated intravascular coagulation (DIC), malignant tumor, hyperlipidemia, diabetes mellitus, nephrotic syndrome, obstructive sleep apnea syndrome (OSAS), coronary heart disease, rheumatic heart disease, infectious endocarditis, dilated cardiomyopathy, hypertensive disease, congenital heart disease, chronic obstructive pulmonary disease, pulmonary 
heart disease, or history of surgery $(10,11)$. Therefore, we speculated that recurrent episodes of PSVT caused the left ventricular thrombus.

The mechanism of thrombus formation by PSVT is unknown, and several factors are potentially involved. Both the onset and termination of PSVT are characteristically abrupt. The sudden attack and cessation of PSVT may result in the loss of effective contraction and relaxation in the atrium and atrioventricular node, leading to the decline or loss of pumping function. The sudden termination of longer duration PSVT attacks may lead to a decline of atrial and atrioventricular nodes' systolic function and then cause turbulent blood flow in the ventricle. This would, in turn, increase the contact between platelets and endothelium; increased platelet activation results in an increased risk of thrombus formation (12-14).

Atrial fibrillation is the most common cause of blood clots in adult arrhythmias; thus, anticoagulant therapy is the routine treatment for such patients. In atrial fibrillation, local blood stasis and turbulent flow give rise to thrombosis formation (14). Atrial fibrillation in PSVT patients is significantly higher than that of the general population and may reach $12-44 \%(15,16)$. In the present case, it is unknown whether the patient developed thrombosis due to the long-term PSVT alone or atrial fibrillation.

In conclusion, this was a rare case of left ventricular thrombus, and cerebral infarction after sustained episodes of PSVT in an infant diagnosed based on cross-sectional echocardiography. We recommend echocardiographic evaluation of the left ventricular function, including the screening for thrombi, and that the possibility of a thrombus must be considered when an abnormal structure is identified in the left ventricle.

The clinical significance of this case report highlights the importance of health education for general community populations. Additionally, the attending parent or guardian should pay adequate attention to abnormal clinical manifestations such as tachypnea, pallor, cyanosis, and poor appetite from the patients' side. If the abovementioned abnormal symptoms occur, the parent or guardian should promptly take their baby to the hospital. From the pediatricians' side, they should be trained for early recognition of the nonspecific clinical manifestations of PSVT, and for making diagnosis by ECG, evaluation of cardiac function and thrombosis by echocardiography, and termination PSVT as rapidly as possible. Thromboprophylactic therapy should be provided in instances of recurrent attacks that are not easily terminated.
In order to prevent this rare but severe clinical complication, future research may concentrate on risk factors leading to thrombosis, including the duration and frequency of the PSVT attack, ventricular rate at the time of the attack, as well as the platelet count and function, thrombin, fibrinolytic enzymes, and so on. More attention should be paid to children with high risk factors, and preventive measures must be taken swiftly against severe complications.

\section{Acknowledgments}

The authors thank the patient's guardian for their cooperation.

Funding: This paper was supported by the Beijing Hospital Administration "Peak Climbing" Talents Development Program (DFL20181301) and the Key Project of Capital Clinical Characteristic Application Research (Z181100001718189).

\section{Footnote}

Reporting Checklist: The authors have completed the CARE reporting checklist. Available at http://dx.doi.org/10.21037/ apm-20-1797

Conflicts of Interest: All authors have completed the ICMJE uniform disclosure form (available at http://dx.doi. org/10.21037/apm-20-1797). Dr. DL reports grants from Beijing Hospital Administration "Peak Climbing" Talents Development Program, grants from Key Project of Capital Clinical Characteristic Application Research, during the conduct of the study. Dr. MMZ reports grants from Beijing Hospital Administration "Peak Climbing" Talents Development Program, grants from Key Project of Capital Clinical Characteristic Application Research, during the conduct of the study. Dr. XHL reports grants from Beijing Hospital Administration "Peak Climbing" Talents Development Program, grants from Key Project of Capital Clinical Characteristic Application Research, during the conduct of the study. Dr. LS reports grants from Key Project of Capital Clinical Characteristic Application Research, during the conduct of the study. Dr. Yao L reports grants from Key Project of Capital Clinical Characteristic Application Research, during the conduct of the study. Dr. Yang L reports grants from Key Project of Capital Clinical Characteristic Application Research, during the conduct of the study. The authors have no other conflicts of interest to 
declare.

Ethical Statement: The authors are accountable for all aspects of the work in ensuring that questions related to the accuracy or integrity of any part of the work are appropriately investigated and resolved. All procedures performed in studies involving human participants were in accordance with the ethical standards of the institutional and/or national research committee(s) and with the Helsinki Declaration (as revised in 2013). This study was approved by the ethical review committee of The Children's Hospital Capital Institute of Pediatrics (registration number: SHERLL2018020). Written informed consent was obtained from the patient for publication of this manuscript and any accompanying images.

Open Access Statement: This is an Open Access article distributed in accordance with the Creative Commons Attribution-NonCommercial-NoDerivs 4.0 International License (CC BY-NC-ND 4.0), which permits the noncommercial replication and distribution of the article with the strict proviso that no changes or edits are made and the original work is properly cited (including links to both the formal publication through the relevant DOI and the license). See: https://creativecommons.org/licenses/by-nc-nd/4.0/.

\section{References}

1. Joung B, Lee M, Sung JH, et al. Pediatric radiofrequency catheter ablation: sedation methods and success, complication and recurrence rates. Circ J 2006;70:278-84.

2. Paul T, Bertram H, Bökenkamp R, et al. Supraventricular tachycardia in infants, children and adolescents: diagnosis, and pharmacological and interventional therapy. Paediatr Drugs 2000;2:171-81.

3. B AG, Mathew RC. Left atrial thrombus in a neonate with normal heart after sustained supraventricular tachycardia. J Clin Diagn Res 2014;8:PD01-2.

4. Russo G, Trappan A, Benettoni A. Unusual left atrial appendage thrombus in a neonate with normal heart

Cite this article as: Li D, Guo J, Li XH, Liao Y, Zhang MM, Shi L, Lin Y, Liu Y. A case report of an infant after episodes of paroxysmal supraventricular tachycardia with left ventricular thrombosis and cerebral infarction. Ann Palliat Med 2021;10(7):8322-8327. doi: 10.21037/apm-20-1797 after sustained supraventricular tachycardia. Int J Cardiol 2008;131:e17-9.

5. Hanséus K, Björkhem G. Left ventricular thrombosis during infancy: report of two cases. Pediatr Cardiol 1995;16:182-5.

6. Salerno JC, Seslar SP. Supraventricular tachycardia. Arch Pediatr Adolesc Med 2009;163:268-74.

7. Tavera MC, Bassareo PP, Neroni P, et al. Supraventricular tachycardia in neonates: antiarrhythmic drug choice dilemma. J Matern Fetal Neonatal Med 2010;23 Suppl 3:30-3.

8. Lupoglazoff JM, Denjoy I. Practical attitude toward arrhythmia in the neonate and infant. Arch Pediatr 2004;11:1268-73.

9. Kantoch MJ. Supraventricular tachycardia in children. Indian J Pediatr 2005;72:609-19.

10. Blann AD, Steele C, McCollum CN. The influence of smoking and of oral and transdermal nicotine on blood pressure, and haematology and coagulation indices. Thromb Haemost 1997;78:1093-6.

11. Nobili L, Schiavi G, Bozano E, et al. Morning increase of whole blood viscosity in obstructive sleep apnea syndrome. Clin Hemorheol Microcirc 2000;22:21-7.

12. Wu N, Tong S, Xiang Y, et al. Association of hemostatic markers with atrial fibrillation: a meta-analysis and metaregression. PLoS One 2015;10:e124716.

13. Lim HS, Willoughby SR, Schultz C, et al. Effect of atrial fibrillation on atrial thrombogenesis in humans: impact of rate and rhythm. J Am Coll Cardiol 2013;61:852-60.

14. Khoo CW, Krishnamoorthy S, Lim HS, et al. Atrial fibrillation, arrhythmia burden and thrombogenesis. Int J Cardiol 2012;157:318-23.

15. Campbell RW, Smith RA, Gallagher JJ, et al. Atrial fibrillation in the preexcitation syndrome. Am J Cardiol 1977;40:514-20.

16. Bauernfeind RA, Wyndham CR, Swiryn SP, et al. Paroxysmal atrial fibrillation in the Wolff-ParkinsonWhite syndrome. Am J Cardiol 1981;47:562-9.

(English Language Editors: J. Jones and J. Chapnick) 